\title{
多孔硅的双峰光致发光谱与光发射机制 ${ }^{*}$
}

\author{
张树霖 ${ }^{(1)}$ 何国山 黄福敏 杨昌黎 \\ (北京大学物理系, 北京 100871; (1) 复旦大学应用表面物理国家重点实验室, 上海 200433) \\ 李经建 才鹏 刘忠范蔡生民 \\ (北京大学化学系光电智能材料研究空, 北京 100871)
}

\section{关键词多孔硅 光致发光徣 光发射机制}

多孔硅的光致发光谱是多孔硅研究的极其重要的一个方面, 除了早已广泛研究的单峰光 致发光谱外,近来有人报道新制备的或经干氧处理的多孔硅在低温下明显存在多峰光致发光 谱 ${ }^{[14]}$, 本文将报道室温下在后处理多孔硅中观察到的双峰光致发光谱, 并讨论后处理条件对光 谱特征的影响, 这种讨论对多孔硅可见光发射机制的探索提供了十分有价值的启示.

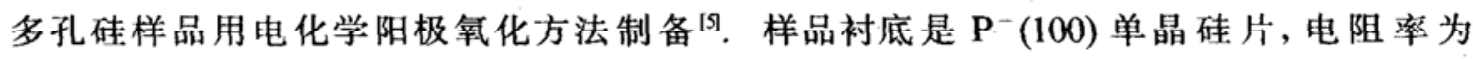
$10 \sim 15 \Omega \cdot \mathrm{cm}$, 电解液是氢绨酸 $(\mathrm{HF})$ 加乙醇的水溶液, 各样品形成时的氢氧酸浓度 $C_{\mathrm{HF}}$ (重 量百分数)、电流密度 $\mathrm{J} / \mathrm{mA} \cdot \mathrm{cm}^{-1}$ 和氧化时间 $t / \mathrm{min}$ 等参数值用加在样品号后面的符号 $(C / J / t)$ 表示. 光致发光谱在 SPEX-1403 双单色仪上测量, 光源为 $457.9 \mathrm{~nm}$ 线的氯离子激 光, 人射激光束用柱型透镜聚焦以消除热效应, 用致冷光电倍增管和光子计数器接收光谱信 号. 光谱仪狭缝为 $200 \mu \mathrm{m}$, 步距 $50 \mathrm{~cm}^{-1}$. 本文所载的光致发光谱均经仪器响应曲线校正.

表 1

\begin{tabular}{|c|c|c|c|c|c|c|}
\hline \multirow{3}{*}{ 光橧 } & \multicolumn{3}{|c|}{ 高能峰 } & \multicolumn{3}{|c|}{ 低能峰 } \\
\hline & 峰位 & 峰寨 & 强度比 & 峰位 & 峰宽 & 强度比. \\
\hline & $E / \mathrm{eV}$ & $\Delta E / \mathrm{eV}$ & $I$ & $E / \mathrm{eV}$ & $\Delta E / \mathrm{eV}$ & $I$ \\
\hline 1 & & & & 1.97 & 0.302 & 1 \\
\hline 2 & 2.51 & 0.732 & 1.32 & 1.96 & 0.265 & 1 \\
\hline 3 & 2.41 & 0.466 & 0.455 & 1.97 & 0.304 & 1 \\
\hline 4 & & & & 1.99 & 0.355 & 1 \\
\hline 5 & 2.48 & 0.749 & 0.278 & 1.97 & 0.260 & 1 \\
\hline
\end{tabular}

图 1 是样品 PS208 $(12 / 50 / 8)$ 和 PS210(14/50/8) 在相同条件下测得的光致 发光谱 (实线) 和高斯线型 的最小二乘法计算机拟合 谱 (虚线); 为了观察方 便, 本文图中的拟合谱均 做了下移处理。从拟合谱 中得到各谱的峰值能量 $E$,

线宽 $\Delta E$ 和强度比 $I$ (同一光谱高能峰强度与低能峰强度比)如表 1 所列. 从图 1-1 和图 1-2可以 看到, 制备当日的光谱是典型的单峰高斯型光谱, 而在大气中陉存一年后的光谱在高能区出现 新峰, 变成明显的双峰谱. 这种在大气中呁存后出现双峰的行为在其它条件制备下的样品中 也出现, 如图 1-4 和图 1-5 所示. 此外, 我们还发现在激光照射下, 高能峰比低能峰强度更快

1994-10-17 收稿, 1995-05-29 收修改稿

*国家自然科学基金资助项目 


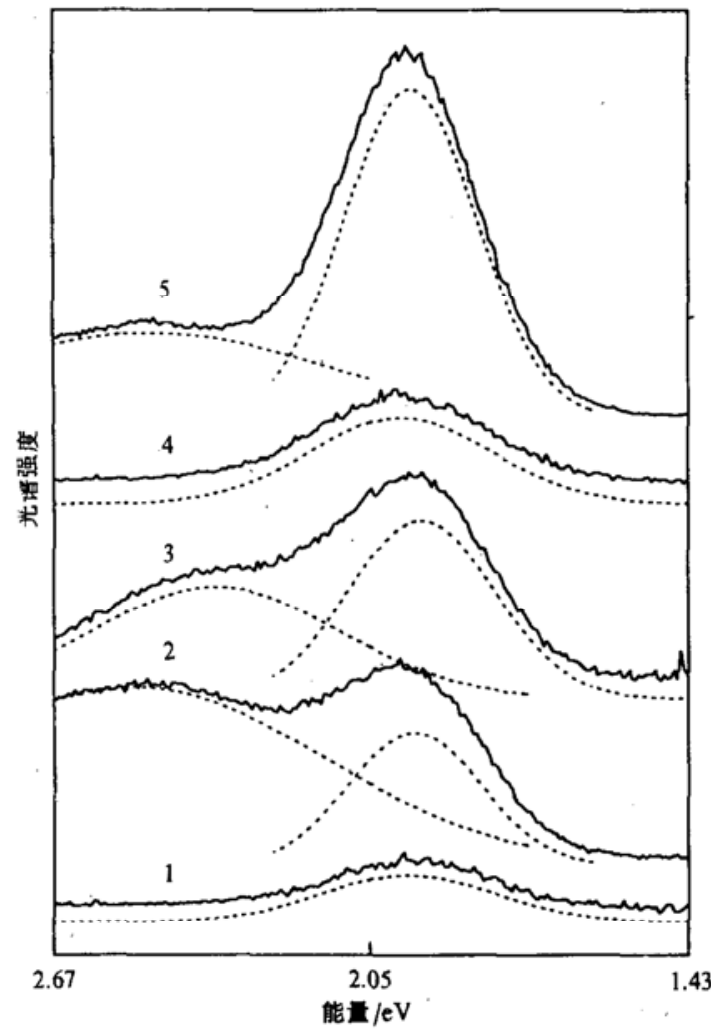

图 1 多孔硅样品的光致发光谱

1 为 PS208(12/50/8) 在制备当日测量的光谱; 2 为 PS208 在 大气中放置一年后测量的光谱; 3 为 PS208存败一年后经 $457.9 \mathrm{~nm}$ 激光照射 $30 \mathrm{~min}$ 后测量的光橧; 4 为 PS210(14/ 50/8) 制备当日测量的光谱; 5 为 PS210 贮存一年后测量 的光谱. 图中虚线橧为计算机拟合谱

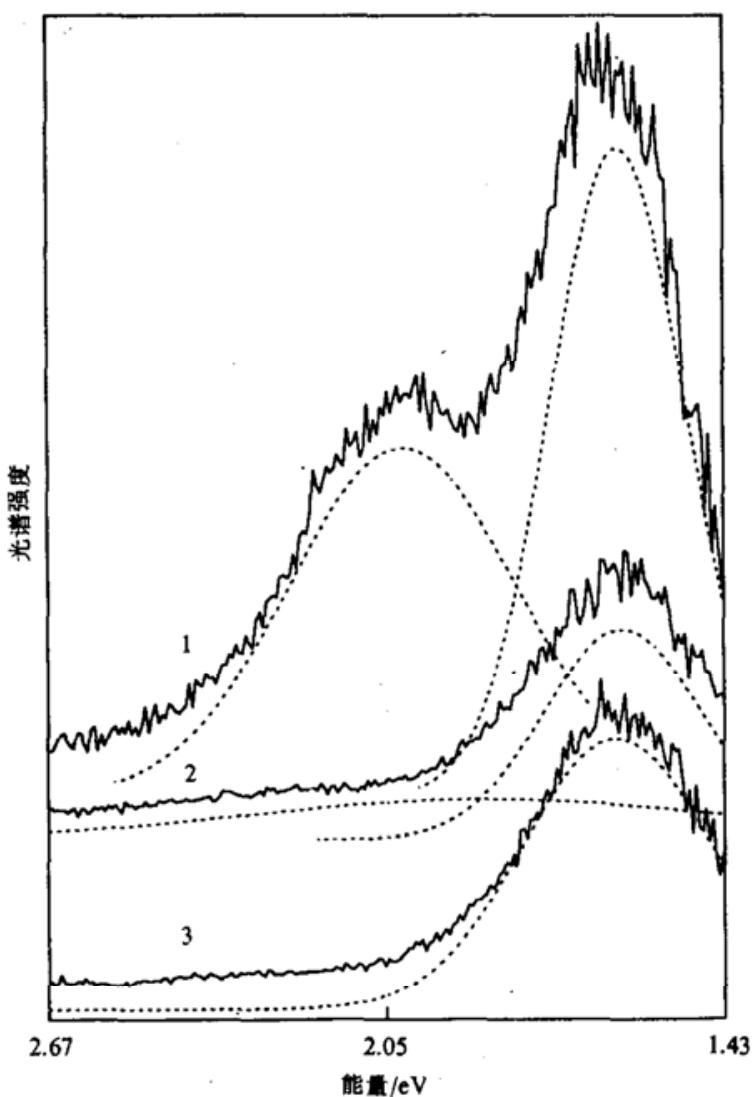

图 2 纯多孔硅薄膜 PS595(33/50/60) 大气中存 贮9d后的显微光致发光谱

1 为激光照射前, 2 为激光照射 $30 \mathrm{~min}$ 后, 3 为激 光照射 $60 \mathrm{~min}$ 后

的下降, 如图 1-3 所示. 由图 1 和表 1 我们还进一步发现两个样品的各个低能峰的峰位能量 $E$ 固定在 $(1.97 \pm 0.02) \mathrm{eV}$, 新出现的高能峰的 $E$ 为 $(2.51 \pm 0.02) \mathrm{eV}$, 激光照射后高能峰的峰值能 量下降至 $2.41 \mathrm{eV}$.

为进一步确证上述现象以及排除双峰现象来自样品中不均匀的不同区域或硅衬底的可能 性, 我们测量了从样品 PS595(33/50/60). 的 Si 衬底上剥落下来的纯多孔硅薄膜的人射激光光斑 约 $1 \mu \mathrm{m}$ 的显微苂光谱,结果示于图 2 和表 2 中. 在图 2-1 中我们看到在大气中存咜 $9 \mathrm{~d}$ 后的

表 2

\begin{tabular}{cccccccc}
\hline & \multicolumn{3}{c}{ 高能峰 } & & \multicolumn{3}{c}{ 低能峰 } \\
\cline { 2 - 5 } \cline { 6 - 8 } 光谱 & 峰位 & 峰宽 & 强度比 & & 峰位 & 峰䆓 & 强度比 \\
& $E / \mathrm{eV}$ & $\Delta E / \mathrm{eV}$ & $I$ & & $E / \mathrm{eV}$ & $\Delta E / \mathrm{eV}$ & 1 \\
\hline 1 & 2.01 & 0.427 & 0.545 & & 1.62 & 0.249 & 1 \\
2 & 1.99 & 0.729 & 0.159 & & 1.61 & 0.297 & 1 \\
3 & & & & & 1.63 & 0.316 & 1 \\
\hline
\end{tabular}

PS 出现了明显的双峰, 图 2-2 和图 2-3 表明, 经激光 照射, 高能峰峰值强度下 降, 照射 $1 \mathrm{~h}$ 后基本消失. 因此图 2 表明在显微光谱 条件下纯多孔硅薄膜的光 


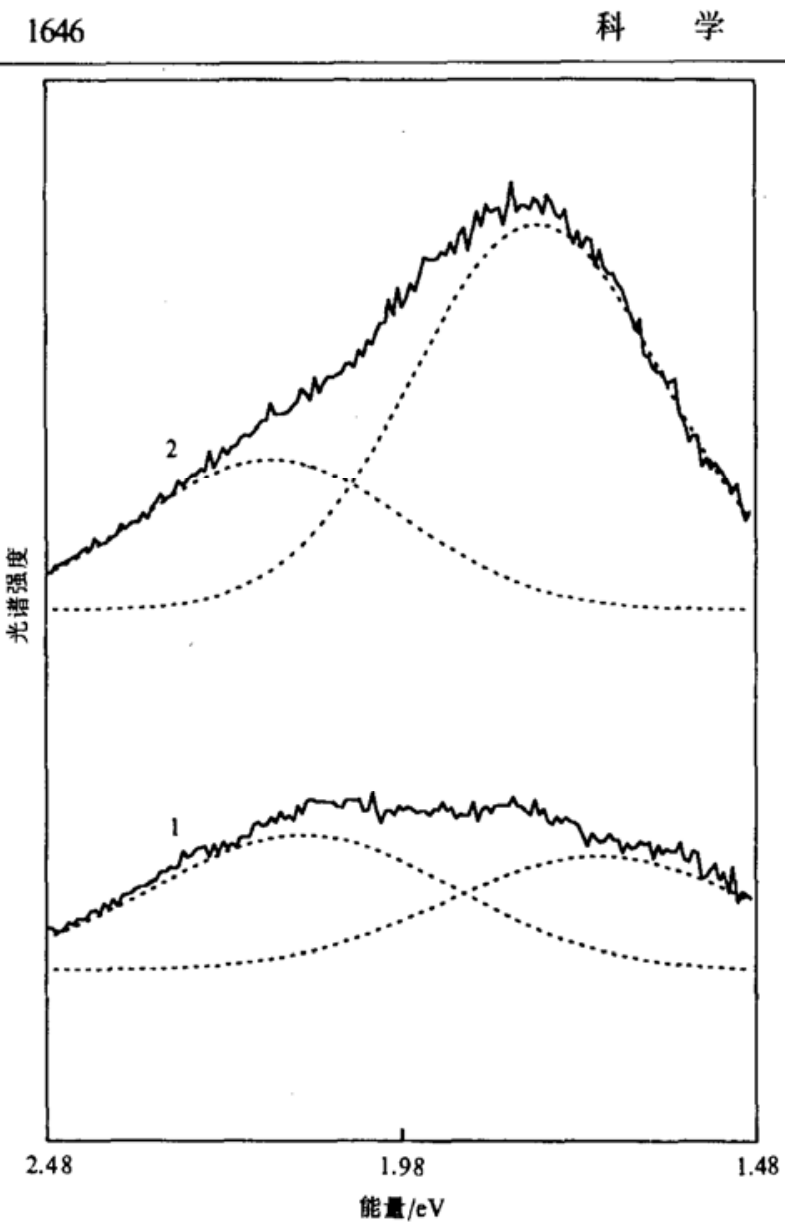

图 3 多孔硅样品 W001(29/50/30) 的光致发光谱 1 为经 20 (重量百分数) $\mathrm{HNO}_{3}$ 溶液浸泡氧化 $70 \mathrm{~min}, 2$ 为 $\mathrm{HNO}_{3}$ 化样品又经 $\mathrm{HF}$ 浸泡还原 $7 \mathrm{~min}$

致发光谱由于形成条件和存咆时间与图 1 样品不同, 因而在峰值能量上与图 1 样品 不一致外, 其余光谱特征与图 1 中的类 似,由此我们可以初步认定:

(1) 图 1 反映的双峰光谱确实来自多 孔硅本身, 且与可能的样品的不均匀无 关;

(2)多孔硅样品经大气中她存一段时 间确实会在新鲜样品原有低能光致发光峰 以外再出现新的高能光致发光峰;

(3) 经激光照射, 高能峰强度相对低能 峰下降得更快;

(4) 考虑到多孔硅样品经柱型透镜焦 照射, 由激光引起的温升效应可以忽略和 $100 \mathrm{~mW}$ 激光经显微光路聚焦将有极大温 升的实验报道 ${ }^{6}$, 以及图 1 和图 2 中高能峰 经激光照射以同样倾向变化的事实, 可以 认为高能峰强度的下降不是热效应引起, 而是光照作用的结果.

据我们所知, 这种由大气存根”起的 双峰光致发光谱及其光致退化的现象至今 尚末见诸报道.

关于上述双峰的出现和退化机制, 我们认为, 多孔硅表面的氧化和去氧化起了重要作用. 二次离子质谱分析也确实表明沐, 室温大气存咜使得新制备的多孔硅的氢化物表面转化成了 混有氧化物的表面, 被大量的 Si-H (或氢氧化合物 ) 所覆盖的新制备的多孔硅, 经在大气中存 败, 在表面形成含 $\mathrm{Si}-\mathrm{O}$ 或 $\mathrm{Si}-\mathrm{H}-\mathrm{O}$ 的化学物质, 因而高能峰极可能就是这些含氧 $\mathrm{Si}$ 化物的发 光峰, 根据光化学理论, 多孔硅在激光照射下, 发生光电化学作用, 在含有水蒸汽的大气中的 主要反应过程是氢取代氧的过程 ${ }^{(8)}$, 在光照下, 多孔硅表面逐渐回复到样品存败前的状态, 于 是因存陉氧化产生的高能峰的退化效应就发生了.

为验证上述分析, 我们测量了经人为化学氧化和还原后处理的多孔硅光致发光谱, 结果示于 图 3 和表 3, 其中图 3-1, 图 3-2分 别是多孔硅 PSW001(29/50/30) 经 20\%(重量百分数) $\mathrm{HNO}_{3}$ 溶 液浸泡氧化 $70 \mathrm{~min}$, 随之又经 $15 \% \mathrm{HF}$ 溶液还原 $7 \mathrm{~min}$ 以后的光致发光谱. 在图 3 中我们看到

表 3

\begin{tabular}{|c|c|c|c|c|c|c|}
\hline \multirow{3}{*}{ 光谱 } & \multicolumn{3}{|c|}{ 高能峰 } & \multicolumn{3}{|c|}{ 低能峰 } \\
\hline & 峰位 & 峰宽 & 强度比 & 峰位 & 峰宽 & 强度比 \\
\hline & $E / \mathrm{eV}$ & $\Delta E / \mathrm{eV}$ & $I$ & $E / \mathrm{eV}$ & $\Delta E / \mathrm{eV}$ & $I$ \\
\hline 1 & 2.12 & 0.371 & 1.08 & 1.74 & 0.390 & 1 \\
\hline 2 & 2. 10 & 0.434 & 0.546 & 1.76 & 0.337 & 1 \\
\hline
\end{tabular}

化学氧化后的样品的光谱 图 3-1在 1.74 和 $2.12 \mathrm{eV}$ 处分别出现了类似图 1 和图 2 中的低能峰和高 能峰, 但当氧化样品经 还原处理后(图 3-2), 可 
以看出, 高能峰相对于低能峰极大地减弱了. 此外, 红外光谱测量也表明, 上述人为化学氧化 使表面的氢原子被氧原子或 $-\mathrm{OH}$ 基所取代 ${ }^{\natural 刀}$. 对多孔硅进行热氧化后处理, 也得到了双峰光致 发光谱. 其中高能峰明显地依赖于氧化温度, 且与表面组成相关. 详细结果已另文报道 ${ }^{1)}$. 这些 实验进一步证实了以上关于高能峰出现和退化机制的分析.

与高能峰不同, 在所有上述实验中, 低能峰的能量和强度在大气存拒和人为化学氧化还原 及热氧化过程中都变化不大, 说明该峰与多孔硅表面化学成分没有直接关联, 是来源于多孔 硅中残留硅体的发光峰.

从对上述实验现象的初步分析使我们清楚地看到, P- 型多孔硅除了有源于残留硅体的光 发射峰外, 还同时存在由表面含氧 $\mathrm{Si}$ 化物的光发射峰, 因此, 本文所述的实验结果也告诉人 们: 用单独和孤立的源于残留硅体的量子限制光发射模型不能解释多孔硅发光的全部现象 ${ }^{[2-11]}$.

\section{参考文献}

1 Nishitani $\mathrm{H}$, Nakata $\mathrm{H}$, Ohyama $\mathrm{T}$ et al. Two peaks on photoluminesœenœ spetrum of porous silicon. In: Jiang P, Zheng $\mathrm{H} \mathrm{Z}$ eds. Proceeding of 2 lst International Conference on the Physics of Semiconductors, Beijing. China, Aug 10 14, 1992. Singapore: World Scientific, 1992, 2: 1455

2 Jiang D T, Coulthard H, Sham T K et al. Observations on the surface and bulk luminescence of porous silicon. J Appl Phys, 1993, 74(10): 6335

3 Cheah K W, Chan T, Lee W L et al. Multiple peak photoluminescence of porous silicon. Appl Phys Lett, 1993, 63 (25): 3464

4 Wang X. Luminescence behavior and mechanism of light-emitting porous silicon. Modern Physics Letters, B, 1994, 8 (2): $69 \sim 92$

5 李经建、万 韧,莠生民等. 化学氧化对多孔佳表面态和光致发光的影响. 物理化学学报, 1994, 10(4): 737

6 Chang I M, Pan S C, Chen Y F. Light-induced degradation on porous silioon. Physical Review, B, 1993, 48(12): 8747

7 Canham L T, Houlton M R, leong W Y et al. Atmospheric impregnation of porous silicon at temperature. J Appl Phys, 1991, 70(1): 422

8 科利斯科夫 K B 著. 光电化学太阳能转换. 北京: 科学出版社, 1994

9 Canham L T. Silicon quantum wire array fabrication by electrochemical and chemical dissolution of wafers. Appl Phys Lett, 1990, 57(10): 1046

10 Zhang S L, Ho Kuok-san, Hou Y T et al. Steplike behavior of photoluminesœnce peak energy and formation of p-type porous silicon. Appl Phys Lett, 1993, 62: 642

11 Wang $\mathrm{X}$, Huang $\mathrm{D}$, Ye L et al. Pinning of photoluminescence peak positions for lightemitting porous silicon: an evidence of quantumsize effect. Phys Rev Lett, 1993, 71(8): 1265

1) 李经建, 朱 涛, 蔡生民等. 多孔硅的热氧化与光致发光, 科学通报 (待发表) 